\title{
Holographic detection of AIS real-time signals based on sparse representation
}

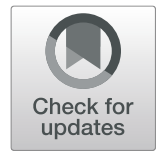

Shuaiheng Huai ${ }^{1}$, Shufang Zhang ${ }^{1 *}$, Jingbo Zhang ${ }^{1}$ and Keyu Huang ${ }^{2}$

\begin{abstract}
To use the existing Automatic Identification System (AIS) shore stations for positioning so that the AIS can be used as an additional land-based positioning system for coastal vessels is a cutting-edge research topic, responding to the call of the International Maritime Organization (IMO). In order to use the ship-borne AIS for positioning function, a holographic detection of AIS real-time signal based on sparse representation is presented in this paper. Considering the working environment and the requirement of AIS real-time signal processing, a novel fast noise resistance Orthogonal Matching Pursuit (OMP) algorithm is presented. Furthermore, the choice and detection of the timestamp of the reconstructed signal is analyzed and carried out which will be used in the ranging system. The experiment results indicate that the proposed fast noise resistance OMP algorithm can greatly reduce the processing time, and the difference in processing time increases with the number of iterations. The improvement in noise immunity is also obvious, and the error rate reduces at about $9 \%$ under the same SNR. The timestamp of the reconstructed signals can be detected successfully. It shows that the holographic detection of AIS real-time signal is achieved satisfactorily.
\end{abstract}

Keywords: Automatic Identification System (AIS), Real-time signal processing, Orthogonal Matching Pursuit (OMP), Noise resistance, Timestamp detection

\section{Introduction}

Positioning, navigation, and timing (PNT) is a key technology to describe time and space. With the development of technology, the dependence on PNT will become bigger and bigger. PNT users may double every 2 years in the next $5-10$ years due to the robotic technology and PNT requirements for other mobile carriers [1]. Nowadays, most PNT users rely on the global navigation satellite systems (GNSS). However, there are certain risks if the GNSS is the only source of PNT information for maritime navigation. The GNSS signal is vulnerable to interferences which may cause signal failure, signal deception, and so on [2-4]. It is a great potential safety hazard. IMO has become aware of the risks and required that all vessels should mandatorily equip land-based backup navigation systems besides the GNSS according to its e-navigation strategy [5-8]. Since the Automatic Identification System (AIS) is the most widely used system in maritime communication field,

\footnotetext{
* Correspondence: sfzhang@dlmu.edu.cn

${ }^{1}$ Information Science and Technology College, Dalian Maritime University,

Dalian 116026, China

Full list of author information is available at the end of the article
}

using the existing AIS as a backup positioning system will save a lot of cost as it has a feasible market application value. Some countries are interested in using the existing AIS shore stations for positioning, with a related new technique called the AIS range (AIS-R) mode. In the European Union, the AIS-R mode is a part of the ACCessibility for Shipping, Efficiency Advantages and Sustainability (ACCSEAS) project which is now mainly operated in the North Sea Region. It concentrates on the feasibility study of AIS-R mode using a combination of DGNSS, AIS, and eLoran which includes the potential signals, geometry, signal strength, and so on $[9,10]$. In China, the related research has been conducted by the Navigation Institute of Dalian Maritime University since 2012 [11-19].

Since the AIS was designed to use as a communication system initially, there are some technical problems that need to be solved before using AIS as a backup positioning system; one of them is the carrier measurement. The carriers in a normal positioning system are dual-phase modulated with the same frequency. However, the carrier of the AIS signal is dual-frequency Gaussian-filtered minimum shift keying (GMSK) modulated according to the 
demands of communication, which is much more complicated. In order to solve the problem of AIS signal carrier measurement, the sparse representation theory was introduced into AIS [18, 19]. The cogitation of signal sparse representation on a redundant dictionary was proposed by Mallat and Zhang in 1993 [20]. The redundant dictionary is one over-complete base functions library. When the signal is represented on the redundant dictionary, only a few base functions have large non-zero coefficients. Thus, the main features of the original signal can be expressed by a few base functions which are also called atoms. The sparse representation can be divided into two parts, the construction of redundant dictionary and the pursuit algorithms. Nowadays, the sparse representation is widely used in image processing which is quite different from real-time processing. We have combined the related research in both fields. The real-time systems have a high requirement for signal processing time, take the AIS as an example, the processing time must be within 1 slot $(26.7 \mathrm{~ms})$.

The AIS-R mode is a ranging system, which is essentially a transmission delay measurement system [21]. Therefore, the ultimate goal is to obtain the time of arrival (TOA) values. The base of a TOA system is to choose the proper timestamps that contain the emission timing information and design the detection method.

\section{Methods}

In this present study, through mathematical formulas transformation of OMP target function and analysis, we improved the OMP algorithm in both noise resistance and processing time. Then two timestamp detection methods of AIS signals were analyzed. As for the simulations, after using the new pursuit algorithm to obtain the sparse representation based on K-SVD to obtain the information of AIS signal within a certain time interval, we presented the timestamp detection of the signal using the differential peak detection and zero-crossing detection. All the simulations were conducted using the MATLAB. This whole process is called the holographic detection of AIS real-time signal. The system block diagram is shown in Fig. 1.

The remainder of this paper is organized as follows. Section 3 introduces the improvements of the OMP algorithm, including the improvement in noise resistance and the improvement in processing time. Section 4 introduces the principle of timestamp and analyzes the detection methods of timestamps. Section 5 provides and discusses the experimental results. Section 6 concludes this paper.

\section{Improved OMP algorithm in AIS}

OMP is a typical greedy algorithm, the basic idea of the algorithm is to select an atom from the dictionary that best matches the signal, construct a sparse approximation, and calculate the residual. Then, continue to select the atoms that best match the signal residual iteratively until the residual is small enough to be negligible. The most important step is to orthogonalize the selected atoms during each iteration. At last, the signal can be represented by the linear combination of these atoms.

\subsection{Principle of OMP}

Assume that the matrix $Y$ represents the signal, $D$ represents the dictionary, and $X$ is the coefficient. The aim of OMP is to find the solution of the following equation

$$
\hat{x}=\underset{x}{\operatorname{Argmin}}\|x\|_{0} \text { s.t. }\|y-D x\|_{2}^{2} \leq \varepsilon
$$

in which $\varepsilon$ represents the error, thus the $\|y-D x\|_{2}^{2} \leq \varepsilon$ is the error constraint. If we turn the error constraint to sparsity constraint, Eq. 1 will be

$$
\hat{x}=\underset{x}{\operatorname{Argmin}}\|y-D x\|_{2}^{2} \quad \text { s.t. }\|x\|_{0} \leq K
$$

in which $K$ is the sparsity and $\|x\|_{0} \leq K$ is the sparsity constraint.

The specific steps of sparsity constraint OMP algorithm are shown as follows:

Input: Dictionary $D$, signal $y$, and target sparsity $K$.

Output: Sparse representation $x$ so that $y \approx D x$.

Initialization: Index set $I=\varnothing$, residual $r=y$, and $x=0$.

1. Calculate the product of the current residual $r$ and the column vector of the dictionary $d^{T}$, find the subscript corresponding to the maximum product, that is

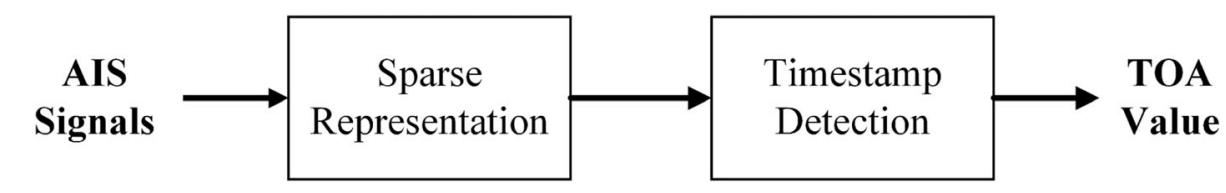

Fig. 1 System block diagram: the simple system block diagram of holographic detection of AIS real-time signals 


$$
\hat{k}=\underset{k}{\operatorname{Argmax}}\left|d_{k}^{T} r\right|
$$

Note that we assume that the columns of $D$ are normalized to unit $\mathrm{l}^{2}$-length for simplicity.

2. Update the index set

$$
I=(I, \hat{k})
$$

3. Use the least square, we can get

$$
x=\underset{x_{I}}{\operatorname{Argmin}}\left\|y-D_{I} x_{I}\right\|_{2}
$$

that is

$$
x_{I}=\left(D_{I}\right)^{+} y
$$

4. Update the residual

$$
r=y-D_{I} x_{I}
$$

The OMP selects the column of $D$ with the highest correlation to the current residual at the first step. Once the column is selected, the signal is orthogonally projected to the selected column at the third step. The residual is recomputed at the fourth step, and the process repeats until the stopping criterion is met.

\subsection{Improvement in noise resistance}

The accuracy of the sparse representation of the AIS signal has a direct impact on the following timestamp detection which is the most important part to obtain the PNT. Considering the working environment of AIS, the noise resistance of the OMP algorithm is really important, and with a better noise resistance means the devices can adapt to harsher environments.

Assume an ideal signal $y$, a white Gaussian noise v, with standard deviation $\sigma$. The signal with noise $z$ is

$$
z=y+v
$$

Then, Eq. (1) will be

$$
\hat{x}=\underset{x}{\operatorname{Argmin}}\|x\|_{0} \text { s.t. }\|z-D x\|_{2}^{2} \leq \hat{\varepsilon}
$$

where $\hat{\varepsilon}$ is dictated by $\varepsilon$ and $\sigma$. If we turn the constraint $\|z-D x\|_{2}^{2} \leq \hat{\varepsilon}$ to a penalty item, then

$$
\hat{x}=\underset{x}{\operatorname{Argmin}}\|z-D x\|_{2}^{2}+\mu\|x\|_{0}
$$

where $\mu\|x\|_{0}$ is the added penalty item. The two equations are equivalent for a proper choice of $\mu$. This equation can also be used as a mathematical model of the pursuit algorithm; although it seems hard to solve, the matching pursuit and basis pursuit algorithms can be used effectively to get approximation solutions [22-24]. To raise the noise resistance is equivalent to reducing the noise $v$, the simplest and most efficient method is to reduce the value of $\|z-y\|_{2}^{2}$. If we split the signal into segments and assume that every segment belongs to the model shown by Eq. (10), then a natural generalization of the model will be

$$
\begin{aligned}
\left\{\hat{x}_{\mathrm{ij}}, \hat{Y}\right\}= & \underset{x_{\mathrm{ij}}, Z}{\operatorname{Arg} \min } \lambda\|Z-Y\|_{2}^{2}+\sum_{\mathrm{ij}} \mu_{\mathrm{ij}}\left\|x_{\mathrm{ij}}\right\|_{0} \\
& +\sum_{\mathrm{ij}}\left\|D x_{\mathrm{ij}}-R_{\mathrm{ij}} Y\right\|_{2}^{2} .
\end{aligned}
$$

In this equation, the first item acts as the global force that demands the proximity between $z$ and $y$ which means to minimize the noise, $\lambda$ is the Lagrange multiplier. The second and the third items make sure that every segment $x_{\mathrm{ij}}$ has a sparse representation with error constraint. $R_{\mathrm{ij}}$ in the third item is the matrix that extracts segments, similar conversion has been practiced in [25].

In Eq. (11), we assume that the dictionary $D$ is known, then there are two unknown variables: the sparse representation $\hat{x}_{\mathrm{ij}}$ for every segment and the overall output $Y$. In order to solve these, we initialize with $Y=Z$ and then seek the optimal $\hat{x}_{\mathrm{ij}}$. Thus, the whole task is decoupled; the form of every segment is

$$
\hat{x}_{\mathrm{ij}}=\operatorname{Arg} \min _{x} \mu_{\mathrm{ij}}\|x\|_{0}+\left\|D x-y_{\mathrm{ij}}\right\|_{2}^{2} .
$$

Note that this is the same form as Eq. (10) which means it can be easily solved by OMP. The second penalty item can be turned into a constraint, then the choice of $\mu_{\mathrm{ij}}$ is handled implicitly. When all $\hat{x}_{\mathrm{ij}}$ are known, return to Eq. (11),

$$
\hat{Y}=\operatorname{Arg} \min _{y} \lambda\|Z-Y\|_{2}^{2}+\sum_{\mathrm{ij}}\left\|D \hat{x}_{\mathrm{ij}}-R_{\mathrm{ij}} Y\right\|_{2}^{2}
$$

needs to be solved. This is a quadratic term that has a closed-form solution of the form 


$$
\hat{Y}=\left(\lambda I+\sum_{\mathrm{ij}} R_{\mathrm{ij}}^{T} R_{\mathrm{ij}}\right)^{-1}\left(\lambda Y+\sum_{\mathrm{ij}} R_{\mathrm{ij}}^{T} D \hat{\mathrm{x}}_{\mathrm{ij}}\right)
$$

in which the matrix to invert is a diagonal matrix, and this calculation can be also done on an atom-to-atom basis [26].

Therefore, by splitting the noisy signals into segments and changing the penalty items, the noise resistance of the OMP is improved.

\subsection{Improvement in processing time}

The AIS is a real-time system, the signal processing time has strict requirements. According to the recommendation proposed by the International Telecommunication Union (ITU), the processing time must be limited in one slot, that is $26.7 \mathrm{~ms}$ [27]. Since we add one more procedure in the previous section to improve the noise resistance of OMP which will definitely increase the processing time, a novel fast OMP processing method is proposed in this section.

From Section 2, we can get that during the greedy selection at each iteration, there is no need to know $r$ or $x$ explicitly, but only $D^{T} r$. Therefore, a lower cost computation of $D^{T} r$ can be used instead of the explicit computation of $r$. Assume $\beta=D^{T} r, \beta^{0}=D^{T} x$ and $T=D^{T} D$, then combine with Eq. (6) and (7), we can get

$$
\begin{aligned}
\beta & =D^{T}\left(y-D_{I}\left(D_{I}\right)^{+} y\right)=\beta^{0}-T_{I}\left(D_{I}\right)^{+} y \\
& =\beta^{0}-T_{I}\left(T_{I, I}\right)^{-1} \beta_{I}^{0} .
\end{aligned}
$$

It means that $\beta$ can be obtained during each iteration without explicit computation of $r$, if $\beta^{0}$ and $T$ are pre-computed. Also, the matrix $T_{I}$ is used instead of applying the complete dictionary $D$. Both of the pre-compute operations will dramatically reduce the processing time.

In summary, by combining the two improvements in noise resistance and processing time, we propose a novel fast noise resistance OMP algorithm. The simulation results will be shown and discussed in Section 4 .

\section{Timestamp detection of AIS real-time signals}

The basic principle of a ranging system is to compute the distance $d$ by measuring the signal transmission delay $\tau$

$$
d=c \tau=c\left(t_{\mathrm{r}}-t_{\mathrm{e}}\right) .
$$

$t_{\mathrm{e}}$ is the emission time and $t_{\mathrm{r}}$ is the receiving time. This is known as the TOA method. In practical application, it is impossible that the receiver knows the emission time accurately, there is always a system clock error in Eq. (16), so the computed distance $d$ is called the pseudorange.

\subsection{Principle of timestamp}

In TOA system, a symbol that contains the emission time information is called timestamp. Detecting timestamps is the key technique in the system. The carriers currently used in TOA system are all dual-phase modulated, so the timestamps can be detected in an arbitrary carrier phase or code phase. However, according to the demands of communication, the carrier of AIS was designed to be dual-frequency GMSK modulated. It is impossible to measure an arbitrary code phase, thus the timestamp in AIS must have some easily detected features. Dr. Johanson has proposed a correlation timestamp detection method based on a fixed message format [10], however, its disadvantage is that the bandwidth resource which is very limited in the AIS is wasted. Since the time division multiple address (TDMA) networking technology is used in the AIS which means that the initial transmission time of each frame can be determined. So the launch time of every bit-0-phase in a frame can be calculated [17]. The bit-0-phase corresponds to the hopping edge as shown in Fig. 2 which are easy to detect, so the bit-0-phase can be regarded as the timestamps in AIS.

Since the reproductive signal in the receiver will change the Gaussian baseband waveform as shown in Fig. 2 before it obtains the binary sequence by bit decision algorithm which only focuses on the bit error rate not the emission time information of bit-0-phase, the research subject should be the Gaussian baseband waveform. The characteristics of the Gaussian baseband waveform are determined by the Gaussian filter and the unit impulse response of the Gaussian filter is expressed as

$$
h(t)=\frac{k}{\sqrt{\pi}} \exp \left(-k^{2} t^{2}\right)
$$

where $k=\sqrt{\frac{2}{\ln 2}} \pi B$ is a parameter related to the Gaussian filter 3-dB bandwidth $B$. The product of $B$ and the input symbol width $T$ are usually considered as the main parameter for designing a Gaussian filter, denoted as $B T$. The unit step response of the Gaussian filter is

$$
s(t)=\int_{-\infty}^{t} h(x) d x=\frac{1}{2}[1+\operatorname{erf}(k t)]
$$

In [17], we have discussed in details that the median point of the Gaussian baseband waveform corresponds to the binary sequence bit-0-phase which can be regarded as the timestamp.

\subsection{Timestamps detection method}

Since the amplitude midpoint corresponds to the digital message sequence bit-0-phase, the amplitude of baseband 

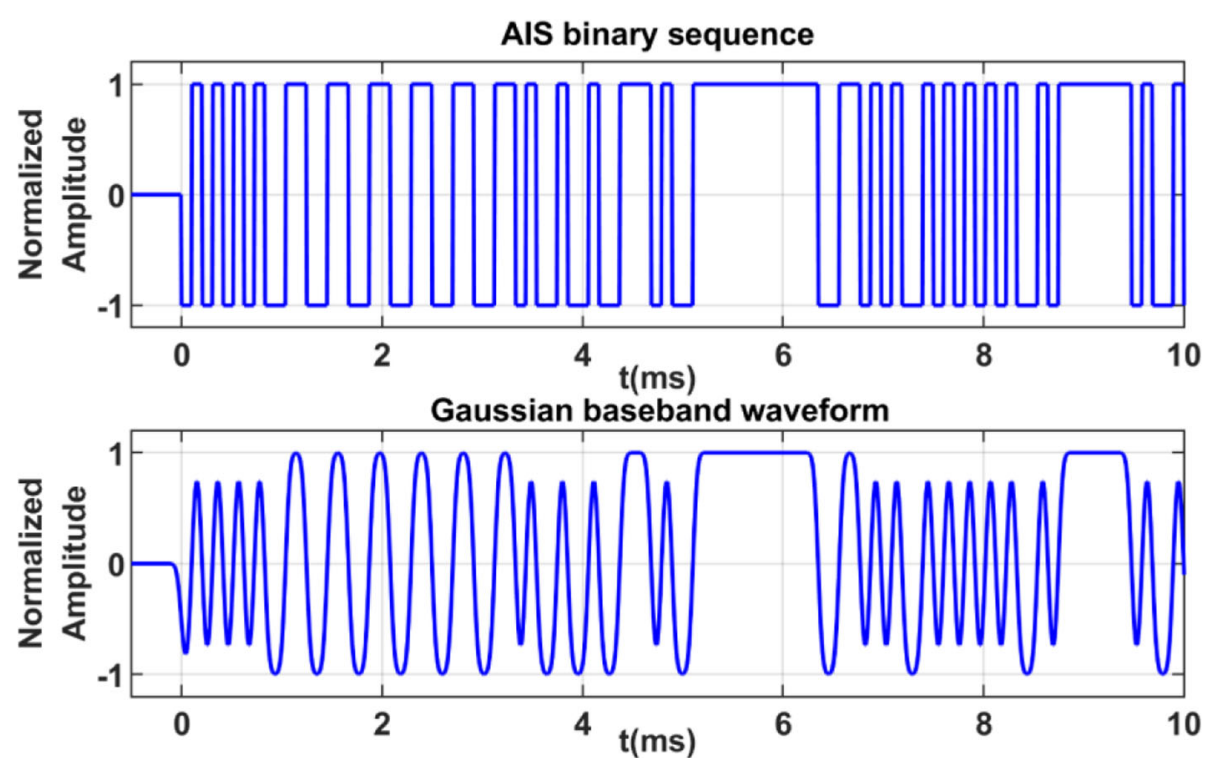

Fig. 2 AIS binary sequence and Gaussian baseband waveform: The upper subfigure is the AIS binary sequence and the bottom subfigure is the Gaussian baseband waveform. The $x$-axis denotes the time in milliseconds, and the $y$-axis is the normalized amplitude. The figure shows that the hopping edge in the binary sequence corresponds to the bit-0-phase in the Gaussian baseband waveform

waveform determines the threshold. In AIS, the binary message sequence can be expressed as

$$
i(t)=2 u(t)-1
$$

where $u(t)$ represents the unit step signal. From Eq. (18) and (19), we can get the corresponding output as

$$
o(t)=2 s(t)-1=\operatorname{erf}(k t)
$$

As shown in Fig. 3, the midpoint of the Gaussian error function is zero. Thus, in an ideal case, the threshold of the detected timestamp is set to be zero. This timestamp detection method is called zero-crossing detection.

The ideal Gaussian baseband waveform rising edge function is defined by the Gaussian error function with the expression of

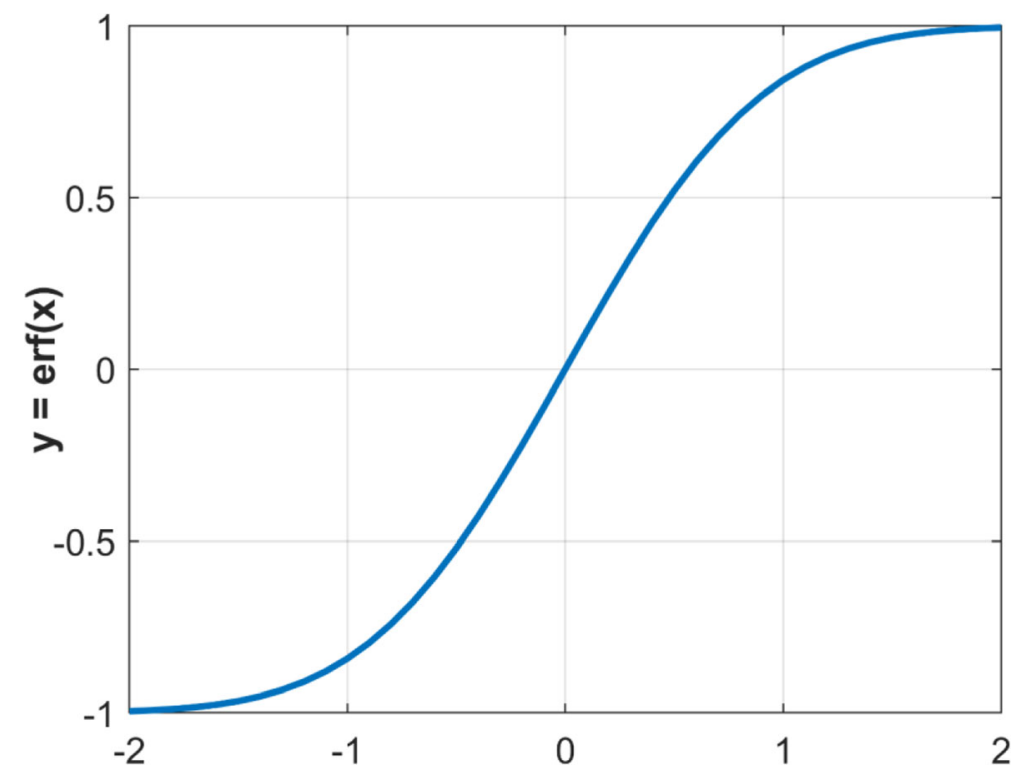

Fig. 3 Gaussian error function waveform: the waveform of Gaussian error function whose midpoint is zero 


$$
\operatorname{erf}(k t)=\frac{2}{\sqrt{\pi}} \int_{0}^{k t} e^{-\eta^{2}} d \eta
$$

Its derivative is expressed as

$$
f(t)=\frac{d}{d t} \operatorname{erf}(k t)=\frac{k}{\sqrt{\pi}} e^{-k^{2} t^{2}} .
$$

According to the definition of the derivative, the differential peak point of it corresponds to the bit-0-phase of the digital sequence. Therefore, this timestamp detection method is called differential peak detection.

\section{Results and discussion}

In this section, first, we assess the performance of the improved OMP algorithm in AIS via numerical simulations. According to [27], the data part of the default transmission packet is 168 bits. Considering this, 64 data signals with the dimension of 168 are used as training signals in the simulation. The hardware specifications used in this study are Intel Core i7-6700 CPU at $3.40 \mathrm{GHz}$ with 16-GB memory.

Figure 4 compares the time performances of three algorithms: the initial algorithm, the processing speedimproved algorithm, and both the processing speed and noise resistance-improved algorithm. Due to the requirements of real-time, the processing time must be within one slot. The black dash line corresponds to the time of one slot in AIS that is $26.7 \mathrm{~ms}$. From the figure, we can see that both of the improved algorithms greatly improve the processing speed. Despite the added denoising process, the processing time of the improved algorithm is still much less than the original one. Furthermore, the slopes of the two lines that represent the two improved algorithms are nearly the same, which means that the time consumption of de-noising process is basically fixed, it does not increase with the increase of iteration. In fact, only when the number of iteration reaches 50 , the processing time of the improved algorithm is about the time of one slot. However, according to [18], the increase in the number of iterations does not improve the accuracy significantly; there is no need to set a big number of iteration.

Setting the number of iterations to 6 , the error rates of three methods are shown in Table 1 . The simulation was repeated 500 times for each condition. In the condition of the same SNR, the error rate of the time improved algorithm is a little bigger than that of the original algorithm. However, once the de-noising process was added, the error rate reduced greatly. For the three algorithms, when the SNR reaches about $15 \mathrm{~dB}$, the error rates come to a threshold.

In Fig. 5, when the number of iteration is the same, the error rate of the time and noise resistance-improved algorithm is far smaller than that of the original algorithm. The error rate of the improved algorithm reduces around 9\% under the same SNR compared to the original algorithm. For the improved algorithm, the number of iterations does not have a great impact on the error rate. The threshold of the error rate of the improved algorithm

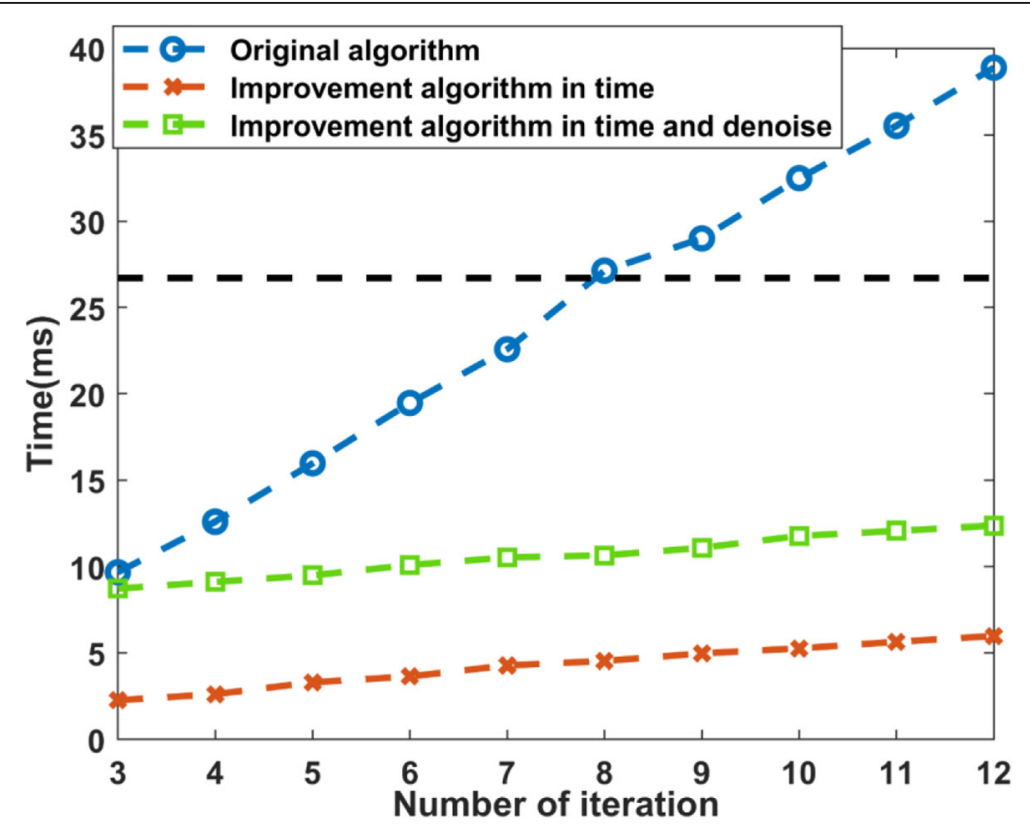

Fig. 4 Time performances of three algorithms: the time performances of the original algorithm (represented by the blue dashed line), the processing time-improved algorithm (represented by the red dashed line), and the processing time and noise resistance-improved algorithm (represented by the green dashed line). The $x$-axis is the number of iterations and the $y$-axis denotes the time in milliseconds 
Table 1 Error rate comparison

\begin{tabular}{llll}
\hline SNR/ & Error rate & & \\
\cline { 2 - 4 } $\mathrm{dB}$ & Original algorithm & Time-improved algorithm & Time and noise resistance-improved algorithm \\
\hline 0 & $11.5 \%$ & $12.3 \%$ & $1.2 \%$ \\
5 & $9.4 \%$ & $10.5 \%$ & $1.2 \%$ \\
10 & $7.8 \%$ & $9.2 \%$ & $1.0 \%$ \\
15 & $7.2 \%$ & $8.8 \%$ & $0.9 \%$ \\
20 & $6.9 \%$ & $7.9 \%$ & $1.0 \%$ \\
25 & $7.6 \%$ & $8.1 \%$ & $1.0 \%$ \\
30 & $7.1 \%$ & $8.2 \%$ & $0.9 \%$ \\
35 & $7.5 \%$ & $8.0 \%$ & $1.0 \%$ \\
40 & $7.2 \%$ & $8.1 \%$ &
\end{tabular}

is about $1 \%$. This time and noise resistance-improved sparse decomposition algorithm is called fast noise resistance OMP algorithm.

The results of timestamp detection are shown in Fig. 6. Due to the noise interference in the Gaussian baseband waveform, it is necessary to filter out the noises before performing the timestamp detection. The noise superimposed on the Gaussian baseband waveform is consistent with the characteristics of white Gaussian noise. The SNR is set to be $10 \mathrm{~dB}$, and the noises are filtered by using a Gaussian filter with the parameter $B T$ set to be 0.6. Figure $6 a$ is the timestamp detection result of zero-crossing detection, the true values and the detected results are exactly the same. Figure $6 \mathrm{~b}$ is the results of differential peak detection for the same Gaussian baseband waveform. The green circles represent the outliers whose amplitudes are related to the SNR. There are outliers because the differential detection is more sensitive to the signal jitter than zero-crossing detection. In practical applications, the outliers can be retained, ignored, corrected, or replaced, depending on the circumstance. The two timestamp detection methods are compared in

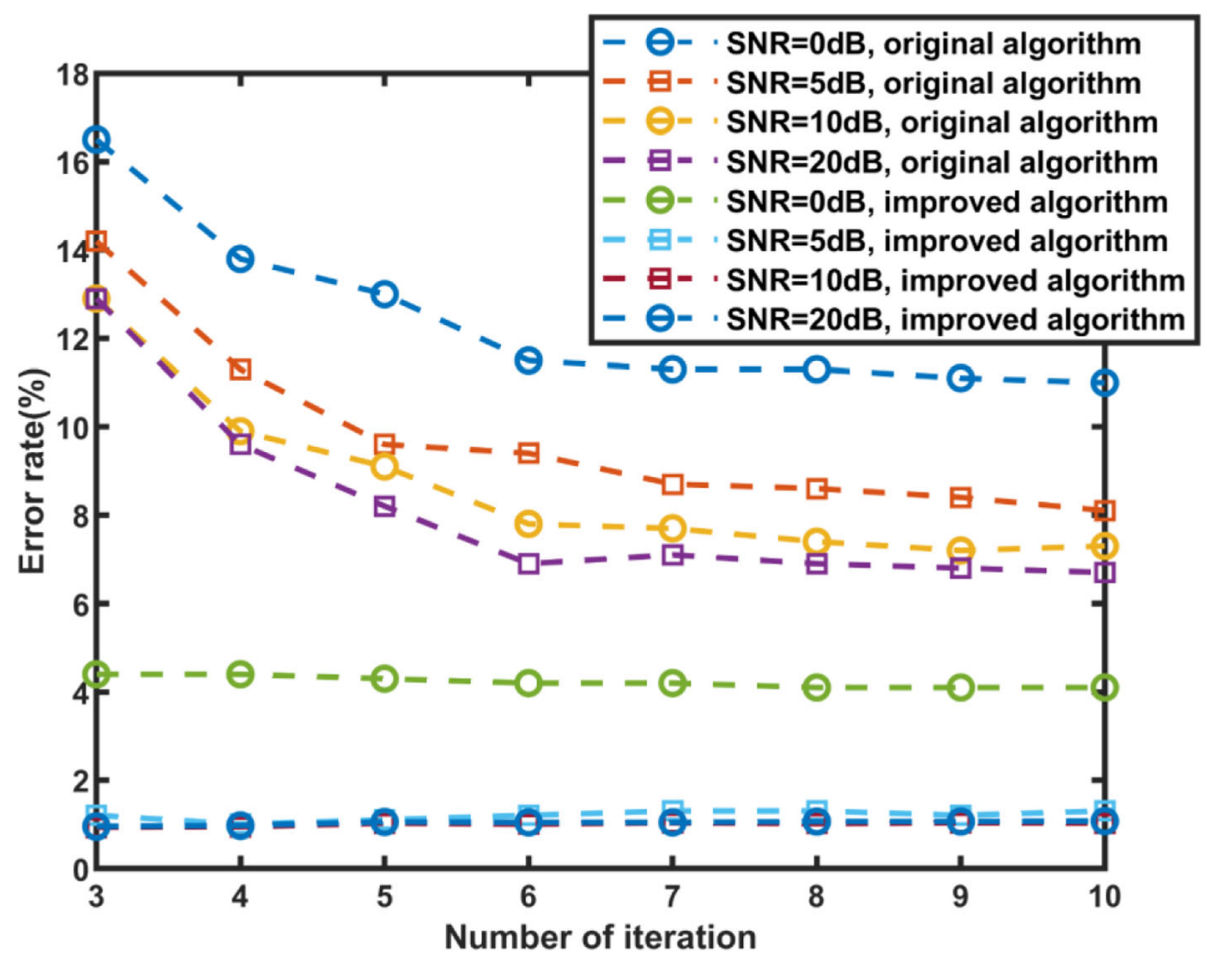

Fig. 5 Error rate under different SNR: the error rate performances of the original algorithm and the processing time and noise resistanceimproved algorithm under different SNRs. The $x$-axis is the number of iterations and the $y$-axis denotes the error rate. The SNR changes from $0 \mathrm{~dB}$ to $20 \mathrm{~dB}$ and the step is $5 \mathrm{~dB}$ 


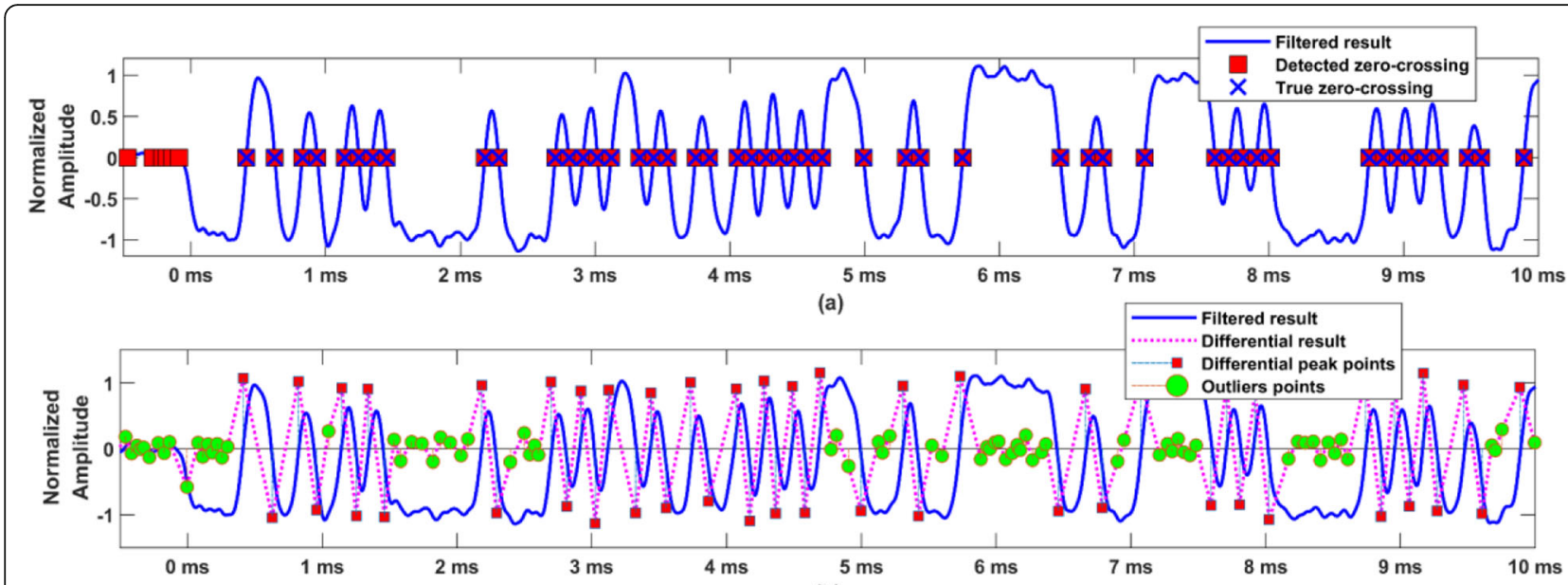

(b)

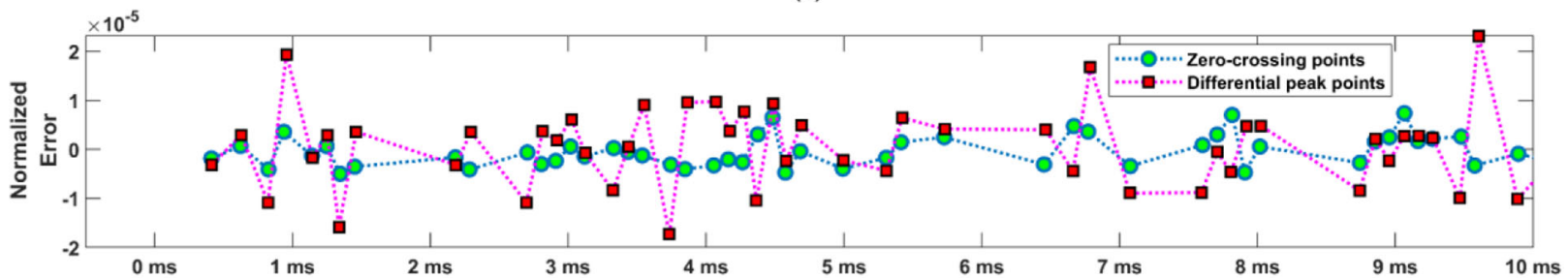

(c)

Fig. 6 Timestamp detection (a), zero-crossing detection (b), and differential peak detection (c). Error distribution: a The detection results of the zero-crossing method. $\mathbf{b}$ The detection results of the differential peak method. Both the $x$-axes denote the time in milliseconds and the $y$-axes are the normalized amplitude. c The comparison of the two methods. The $x$-axis denotes the time in milliseconds and the $y$-axis is the normalized error

Fig. 6c. We can see that the error jitter range of the differential peak detection is bigger than that of the zero-crossing detection.

Based on the above discussions and results, we propose a holographic detection of AIS real-time signals based on the sparse representation. The specific system block diagram is shown in Fig. 7.

\section{Conclusions}

In this paper, the OMP algorithm was improved in both processing speed and noise resistance and applied on the sparse representation of AIS real-time signals. Based on the mathematical analysis and formulas transformation of the OMP target function, we achieved the improvement in the noise resistance of OMP by splitting the noisy signals into segments and changing the penalty items. In addition, the processing speed was accelerated greatly by pre-computing two key parameters. The proposed algorithm was named the fast noise resistance OMP algorithm. The experiment results indicated that the proposed fast noise resistance OMP algorithm can greatly improve both the noise immunity and the processing speed at the same time. The difference in processing time increases with the number of iteration and the error rate reduces around 9\% under the same SNR compared to the original algorithm.

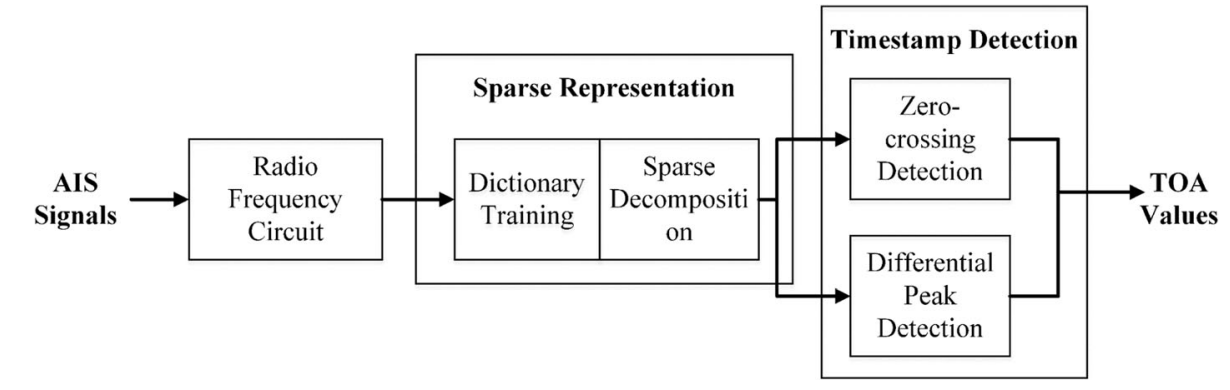

Fig. 7 Holographic detection of AIS real-time signals: the detailed system block diagram of holographic detection of AIS real-time signals 
Considering the GMSK modulation of the AIS signals, the carrier phase cannot be measured like other TOA systems. Through the analysis of the characteristics of AIS, the bit-0-phase was regarded as the timestamp. On the base of Gaussian baseband waveform analysis, two timestamp detection methods, the zero-crossing detection and differential peak detection, were proposed and simulated.

Based on the theoretical analysis and simulated verification of the above two parts, the idea of holographic detection of AIS real-time signals was presented.

The current work is an important part of realizing the positioning function of AIS. For further work, the results of holographic detection can be used in the pseudorange measurement in AIS-R mode. The proposed algorithm will be embedded in the ship-borne AIS equipment to do field tests to be verified and optimized. The theory proposed in the paper provides a reference for real-time signal processing system.

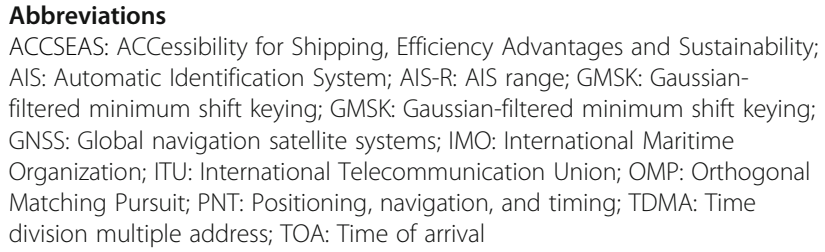
AIS: Automatic Identification System; AIS-R: AIS range; GMSK: Gaussianfiltered minimum shift keying; GMSK: Gaussian-filtered minimum shift keying; GNSS: Global navigation satellite systems; IMO: International Maritime Organization; ITU: International Telecommunication Union; OMP: Orthogonal Matching Pursuit; PNT: Positioning, navigation, and timing; TDMA: Time division multiple address; TOA: Time of arrival

\section{Acknowledgments}

This work was supported by the Chinese National Science Foundation (Nos. 61231006, 61501078), Liaoning Natural Science Foundation (No. 20180550964) and Doctoral Scientific Research Starting Foundation of Liaoning Province (No. 20170520091).

\section{Funding}

Not applicable.

Availability of data and materials

Not applicable.

\section{Authors' contributions}

SH provided the idea, carried out the simulations, and drafted the manuscript. SZ supervised the work and revised the manuscript. JB contributed to the simulations and writing. KY revised the manuscript. All authors read and approved the final manuscript.

\section{Authors' information}

Not applicable.

\section{Competing interests}

The authors declare that they have no competing interests.

\section{Publisher's Note}

Springer Nature remains neutral with regard to jurisdictional claims in published maps and institutional affiliations.

\section{Author details}

'Information Science and Technology College, Dalian Maritime University, Dalian 116026, China. ${ }^{2}$ The Faculty of Electrical Engineering and Information Technology, Institute of Micro and Sensor Systems,

Otto-von-Guericke-University Magdeburg, 39106 Magdeburg, Germany.
Received: 16 January 2019 Accepted: 19 March 2019

Published online: 02 April 2019

\section{References}

1. Y.X. Yang, X.Y. Li, Micro-PNT and comprehensive PNT. Acta Geodaetica et Cartographica Sinica 46(10), 1249-1254 (2017)

2. D. Schmidt, K. Radke, S. Campete, E. Foo, M. Ren, A survey and analysis of the GNSS spoofing threat and countermeasure. ACM Comput. Surv. 48(4), 1-31 (2016)

3. S. Morosi, A. Martinelli, E. Del Re, Peer-to-peer cooperation for GPS positioning. Int. J. Satell. Commun. Netw. 34 (2016)

4. S Jing, XQ Zhan, X Liu, SJ Feng. GNSS vulnerability assessment based on application suitability. Paper presented at the $27^{\text {th }}$ International Technical Meeting of the Satellite Division of the Institute of Navigation, ION GNSS 2014, Tampa, 8-12 September, pp. 2291-2298 (2014)

5. G.T. Becker, S. Lo, D. De Lorenzo, D. Qiu, C. Paar, P. Enge, in Paper presented at the 22nd International Technical Meeting of the Satellite Division of the Institute of Navigation, Savannah USA. Efficient authentication mechanisms for navigation systems-a radio-navigation case study (2009), pp. 2209-2220

6. D. Whelan, P. Enge, G. Gutt, in Paper presented at the $23^{\text {rd }}$ International Technical Meeting of the Satellite Division of the Institute of Navigation, Portland, USA. Robust time transfer from space to backup GPS (2010), pp. 907-914

7. G.H. Kim, S.H. Jeon, C.W. Kim, D.W. Han, C.D. Kee, S.J. Choi, in Paper presented at the Institute of Navigation International Technical Meeting. A combined numerical-emperical orbit propagation algorithm for satellite tracking and backup navigation system (Newport, 2012), pp. 812-819

8. Strategy for the development and implementation of e-navigation, Available online, http://www.imo.org/en/OurWork/Safety/Navigation/Pages/ eNavigation.aspx. Accessed 27 Mar 2019.

9. Y. Jiang, H. Q, K.Z. DK Yang, Map projection positioning method in ranging-mode of automatic identification system. ICIC Express Letter 10, 1093-1100 (2016)

10. G. Johnson, P. Swaszek, J. Alberding, M. Hoppe, J.H. Oltmann, The feasibility of $\mathrm{r}$-mode to meet resilient PNT requirements for e-navigation, Paper presented at the $27^{\text {th }}$ International Technical Meeting of the Satellite Division of the Institute of Navigation, Tampa, USA (2014), pp. 3076-3100

11. Q. Hu, Y. Jiang, S.F. Zhang, Development of an automatic identification system autonomous positioning system. Sensors 15, 28574-28591 (2015)

12. Y. Jiang, S.F. Zhang, D.K. Yang, A novel position estimation method based on displacement correction in AIS. Sensors 14, 17376-17389 (2014)

13. X.Y. Wang, S.F. Zhang, X.W. Sun, The additional secondary phase correction system for AIS signals. Sensors 17, 736 (2017)

14. X.Y. Wang, S.F. Zhang, Evaluation of multipath signal loss for AlS signals transmitted on the sea surface. Ocean Eng. 146, 9-20 (2017)

15. X.Y. Wang, S.F. Zhang, X.W. Sun, Research on the additional secondary phase factor for automatic identification system signals transmitted over a rough sea surface. Sensors 18, 617 (2018)

16. Y. Jiang, J.N. Wu, S.F. Zhang, An improved positioning method for two base stations in AIS. Sensors 18, 991 (2018)

17. J.B. Zhang, S.F. Zhang, J.P. Wang, Pseudorange measurement method based on AIS signals. Sensors 17, 1183 (2017)

18. S.H. Huai, S.F. Zhang, Research on pursuit algorithms for sparse representation of AIS real-time signal. Journal of Dalian University of Technology 58(5), 533-538 (2018)

19. S.H. Huai, S.F. Zhang, A novel sparse representation algorithm for AIS realtime signals. EURASIP J Wirel Commun. Netw. 223 (2018)

20. S. Mallat, Z. Zhang, Matching pursuit with time-frequency dictionaries. IEEE Trans Signal Process. 41(12), 3397-3415 (1993)

21. Y.T. Ma, K. Pahlavan, Y.S. Geng, in Paper presented at the 2014 IEEE $25^{\text {th }}$ Annual International Symposium on Personal, Indoor and Mobile Radio Communication. Comparison of POA and TOA based ranging behaviour for RFID application (Washington DC, 2014), pp. 1722-1726

22. RR Coifman, MV Wickerhauser. Entropy-based algorithms for best basis selection. IEEE Tran. Information Theory. 38, pp. 713-718 (1992)

23. YC Pati, R Rezaiifar, PS Krishnaprasad, Orthogonal Matching Pursuit: Recursive function approximation with applications to wavelet decomposition. Paper presented at the Proc. 27th Asilomar Conf. Signals, Systems and Computers, Pacific Grove, CA, 1-3 November 1993, 1, pp.40-44

24. S.S. Chen, D.L. Donoho, M.A. Saunders, Atomic decomposition by basis pursuit. SIAM Rev. 43(1), 129-159 (2001) 
25. S Roth, MJ Black, Fields of experts: a framework for learning image priors. Paper presented at IEEE Computer Society Conference on Computer Vision \& Pattern Recognition, IEEE, 2005

26. M. Elad, M. Aharon, Image denoising via sparse and redundant representation over learned dictionaries. IEEE Trans. Image Processing 15(12), 3736-3745 (2006)

27. Recommendation ITU-R M.1371-5, Technical Characteristics for an Automatic Identification System Using Time Division Multiple Access in the VHF Maritime Mobile Band. 2014

Submit your manuscript to a SpringerOpen ${ }^{\circ}$ journal and benefit from:

- Convenient online submission

Rigorous peer review

- Open access: articles freely available online

- High visibility within the field

- Retaining the copyright to your article

Submit your next manuscript at $\boldsymbol{\wedge}$ springeropen.com 